\title{
Three selfish spanning tree games
}

\author{
Laurent Gourvès $^{1,2}$ and Jérôme Monnot $^{1,2}$ \\ 1. CNRS, UMR 7024, F-75775 Paris, France \\ 2. Université de Paris-Dauphine, LAMSADE, F-75775 Paris, France \\ \{laurent.gourves, monnot\}@lamsade.dauphine.fr
}

\begin{abstract}
We study a problem in a network. The input is an edgeweighted graph $G=(V, E)$ such that $V$ contains a specific source node $r$. Every $v \in V \backslash\{r\}$ is an entity which wants to be connected to $r$ either directly or via other entities. The main question is how do the entities deviate from a socially optimal network if they are not monitored by a central authority. We provide theoretical bounds on the (strong) price of anarchy of this game. In particular, three variants - each of them being motivated by a practical situation - are studied.
\end{abstract}

\section{Introduction}

How (in)efficiently self-interested agents make use of a common resource? This question is central in computer science because today's major platform - networks - is often operated by spontaneous and selfish users. In recent works including $[1-3]$ the situation is modelled as a strategic game. A strategic game is a tuple $\left\langle N,\left(S_{i}\right)_{i \in N},\left(u_{i}\right)_{i \in N}\right\rangle$ where $N$ is the set of players while $S_{i}$ and $u_{i}$ are respectively the set of strategies of player $i$ and her utility function. Players are supposed to be selfish and rational. Each of them chooses a strategy in order to maximize her own utility.

In this paper, we study three versions of a selfish spanning tree game (SSTgame in short) respectively denoted by min SST-game, max SST-game and bottleneck SST-game. The SST-game is defined upon a complete graph $G=(V, E)$ where every edge $e \in E$ has a positive weight $w(e)$. Each node except a specific source node $r$ is controlled by a self-interested player. Players want to be connected to $r$ either directly or via another player, herself connected to $r$. For the min SST-game (resp. the max SST-game), the utility of a player is the negative of the weight (resp. the weight) of the first edge of the (unique) path between her and $r$. For the bottleneck SST-game, a player's utility is the minimum weight of the edges of the path between her and $r$.

The SST-game is motivated by two situations: when the source node $r$ sends a message to all player nodes (one-to-all communications) and when each player node sends a request to $r$ (all-to-one communications). For the min SST-game, the weight of an edge can represent its cost. If each node pays the price of its upstream edge then the cost of the whole network is covered. Hence each player wishes to receive the message at the lowest price. Another application of the min SST-game arises in power control for static wireless networks. The weight 
of an edge represents its length and the power needed to send a message to a remote node increases with the distance. Hence each player wishes to consume as few power as possible to send its request. For the max SST-game, the weight of an edge represents its reliability: the higher the weight of an edge, the less a message passing through it is vitiated by errors. Then it is in a player's interest to choose the most reliable link from which data arrive in order to spend as few time as possible in repairing it (it is assumed that a message is always sent or forwarded without errors). For the bottleneck SST-game, the weight of an edge represents its free bandwidth. For one-to-all communications, the flow of data at a node depends on the flow at each intermediate node, i.e. it is limited by the edge with smallest free bandwidth. Therefore each player of the bottleneck SST-game wants to maximize her own flow.

The players of the SST-game may not spontaneously reach a social optimum as uncoordinated decisions and conflicting interests often lead to suboptimal performances. The main concern of this paper is to say how (in)efficiently players of the SST-game make use of the graph if they act selfishly. To do so, we study the price of anarchy (PoA) [11] and the strong price of anarchy (SPoA) [2] of the SST-game. These two measures resort to two important concepts in game theory - the Nash equilibrium and the strong equilibrium - to quantify the performance's deterioration due to self-interested behaviors.

\section{Definitions and notations}

The ssT-game. We are given a complete graph $G=(V, E)$ on $n+1$ vertices where $V$ contains a specific node $r$ and a weight function $w: E \rightarrow \mathbb{R}_{+}$. The SST-game is such that $N=V \backslash\{r\}$ (each node except $r$ is a player) and $S_{i}=V$ for all $i \in N$ (each player selects a node). The number of players is finite and denoted by $n$. A strategy profile (or state) $\sigma=\left(\sigma_{1}, \sigma_{2}, \ldots, \sigma_{n}\right)$ is an element of $S=S_{1} \times S_{2} \times \cdots \times S_{n}$. We say that $\sigma$ connects $i \in N$ to the source iff there is a path between $i$ and $r$ in the graph $\left(V,\left\{[u, v]: \sigma_{u}=v\right.\right.$ or $\left.\left.\sigma_{v}=u\right\}\right)$. For the min SST-game, the utility of a player $i$ is defined as $u_{i}(\sigma)=-w\left(\left[i, \sigma_{i}\right]\right)$ if $\sigma$ connects $i$ to $r$, otherwise $u_{i}(\sigma)=-\infty$. For the max ssT-game, the utility is defined as $u_{i}(\sigma)=w\left(\left[i, \sigma_{i}\right]\right)$ if $\sigma$ connects $i$ to $r$, otherwise $u_{i}(\sigma)=-\infty$. For the bottleneck SST-game, the utility is defined recursively as

$$
u_{i}(\sigma)= \begin{cases}w\left(\left[i, \sigma_{i}\right]\right) & \text { if } \sigma_{i}=r \\ \min \left\{w\left(\left[i, \sigma_{i}\right]\right), u_{\sigma_{i}}(\sigma)\right\} & \text { if } \sigma \text { connects } i \text { to } r \\ -\infty & \text { otherwise }\end{cases}
$$

Every player $i$ selects her strategy $\sigma_{i}$ so that $u_{i}(\sigma)$ is maximum. The graph being complete, any player can select her direct link to $r$ and have a utility different from $-\infty$. Therefore the players always build a spanning tree of $G$ if none of them can unilaterally improve her utility.

Along this article, the spanning tree induced by a strategy profile $\sigma$ is denoted by $T^{\sigma}$ and defined as $\left\{[i, j] \in E: \sigma_{i}=j\right.$ or $\left.\sigma_{j}=i\right\}$. The strategy profile induced 
by a spanning tree $T$ is denoted by $\sigma^{T}$ and defined as $\sigma_{i}^{T}=j$ for all $i \in N$ where $[i, j]$ is the first edge of the unique path between $i$ and $r$ in $T$.

Equilibria and the price of anarchy. Given $\sigma \in S$, let us denote by $\left(\left.\sigma\right|_{i} s\right)$ the strategy profile where $\sigma_{i}$ is replaced by $s$ in $\sigma$ whereas the strategy of the other players is unchanged, i.e. $\left(\sigma_{1}, \ldots, \sigma_{i-1}, s, \sigma_{i+1}, \ldots, \sigma_{n}\right)$. A Nash equilibrium is a stable state in which no player has an incentive to unilaterally move away. That is, $\sigma \in S$ is a Nash equilibrium if $\forall i \in N, \forall s \in S_{i}, u_{i}\left(\left.\sigma\right|_{i} s\right) \leq u_{i}(\sigma)$.

We speak about pure Nash equilibria when every player $i$ deterministically chooses a strategy $\sigma_{i} \in S_{i}$. The SST-game always has a pure Nash equilibrium since such a state can be computed as follows. Start with any pure strategy profile (e.g. $\forall i \in N, \sigma_{i}=r$ ) and change the strategy of a player as far as it is profitable (i.e. her utility strictly increases). If such a deviation is not possible then the current state is a Nash equilibrium. Otherwise the utility of at least one player has increased and the utility of all the others has not decreased. Hence if $\sigma$ and $\sigma^{\prime}$ respectively denote the strategy profile before and after the deviation, we have $\sum_{i \in N} u_{i}\left(\sigma^{\prime}\right)>\sum_{i \in N} u_{i}(\sigma)$. One can repeat the process until a pure strategy Nash equilibrium, i.e. a local optimum for $\sum_{i \in N} u_{i}(\sigma)$, is reached.

We only consider pure strategies so we omit from now on the adjective "pure". The price of anarchy (PoA) [11] is the value of the worst Nash equilibrium relative to the social optimum. As usual the social welfare is defined as the sum of the players' utility. We use a function $\mathcal{S}: S \rightarrow \mathbb{R}$ defined as $\mathcal{S}(\sigma)=\sum_{i \in N} u_{i}(\sigma)$.

Let $\sigma$ be a strategy profile at Nash equilibrium whereas $\sigma^{*}$ denotes a strategy profile induced by a social optimum. The PoA of the SST-game is the maximum value of $\mathcal{S}(\sigma) / \mathcal{S}\left(\sigma^{*}\right)$ over all instances.

One particular weakness of the Nash equilibrium is its vulnerability to deviations by coalitions of players. Selfishness does not avoid cooperation as long as it is profitable. Aumann introduced the notion of strong equilibrium [4]. It is a Nash equilibrium where no set of players can cooperatively deviate in a way that benefits all its members, taking the strategies of the players outside the coalition as given. Given two strategy profiles $\sigma, \beta$ and a set $N^{\prime} \subseteq N,\left(\left.\sigma\right|_{N^{\prime}} \beta\right)$ denotes the strategy profile $\alpha$ where $\alpha_{i}=\sigma_{i}$ if $i \in N \backslash N^{\prime}$ and $\alpha_{i}=\beta_{i}$ if $i \in N^{\prime}$. Then $\bar{\sigma}$ is a strong equilibrium if for all $N^{\prime} \subseteq N$, there is no $\beta \in S$ such that $u_{i}\left(\left.\bar{\sigma}\right|_{N^{\prime}} \beta\right)>u_{i}(\bar{\sigma})$ for all $i \in N^{\prime}$. Andelman, Feldman and Mansour [2] proposed the notion of strong price of anarchy (SPoA) which is the PoA restricted to strong equilibria. The SPoA of the SST-game is the maximum value of $\mathcal{S}(\bar{\sigma}) / \mathcal{S}\left(\sigma^{*}\right)$ over all instances.

\section{Contribution and related work}

Contribution. We first show that any Nash equilibrium of the min SsT-game is a strong equilibrium (actually the proof also works for the max SST-game). Since $\mathrm{PoA}=\mathrm{SPoA}$ in this situation we only study the PoA of the min SST-game. We first observe that the PoA of the min SST-game is in general unbounded so we restrict ourselves to instances satisfying the triangle inequality. Under this standard assumption it is proved in Section 4 that $\mathrm{PoA} \in \Theta(\log n)$ and $\mathrm{PoA} \leq d^{*}$ 
where $n$ is the number of players (i.e., $n=|N|=|V|-1$ ) and $d^{*}$ is the depth (maximum number of edges from a leaf to the root) of the tree induced by a social optimum. In addition we bound the PoA with respect to a modified social welfare which is motivated by energy consumption in wireless communications. The PoA of the max SST-game and the bottleneck SST-game are studied in Section 5 (the triangle inequality is not imposed anymore). For the former we prove that $\mathrm{PoA}=1 / \Delta^{*}$ where $\Delta^{*}$ is the maximum degree of a player node (i.e. all nodes except $r$ ) in the tree induced by a social optimum. A tight example shows that the PoA is still $1 / \Delta^{*}$ if the triangle inequality is satisfied. For the bottleneck SST-game we show that any Nash equilibrium is a social optimum $(\mathrm{PoA}=1)$, implying that any Nash equilibrium is a strong equilibrium. Some concluding remarks are given in Section 6. Due to space limitations, some proofs are given in the appendix.

Related work. In 1973 Claus \& Kleitman [6] introduced the problem of allocating the cost of a spanning tree. Subsequently Bird [5] and Granot \& Huberman $[8,9]$ studied the problem as a cooperative game: the players must agree on the structure (the tree) and how its cost is shared. Bird's cost allocation [5] consists in computing a minimum cost spanning tree and each player-node pays the price of the first edge of her path to the source. This allocation belongs to the core ${ }^{1}$ of the game; further results on the core are provided by Granot \& Huberman $[8$, 9]. Then the min SST-game (resp. max SST-game) is a non cooperative version of Claus \& Kleitman's problem with a Bird like allocation rule.

A central network problem viewed as a strategic game is the one introduced by Anshelevich et al. [3] (subsequently studied by Albers [1]). We are given an edge-weighted graph $G=(V, E)$ and pairs $\left(s_{i}, t_{i}\right) \in V \times V$. Each $s_{i}$ wants to connect to $t_{i}$ so the strategies of $i$ are the paths from $s_{i}$ to $t_{i}$ in $G$. The weight $w(e)$ of an edge $e$ represents a cost that is evenly shared among its users. Namely $e$ costs $w(e) / \nu(e)$ to each of its users where $\nu(e)$ denotes the number of $e$ 's users (this cost sharing method is called the shapley cost in the following). The total cost of a player is the sum of the prices she is charged over all edges in her path. Taking the social cost as the sum of all player's individual cost, Anshelevich et al. show that there always exists a Nash equilibrium with social cost at most $O(\log |V|)$ times the social optimum. In the SST-game all $t_{i}$ 's are the same vertex but the main difference resides in the way an agent's utility is defined. In contrast to the shapley cost where a player pays a fraction of each edge of her path, a player's utility in the min SST-game depends only on the first edge. Actually the three variants of the SST-game studied in this paper model situations where the agents' utility is ruled by the topology since quantities like electric power, time (to repair a message) or bandwidth are, unlike money, hard to transfer.

The min SST-game is strongly related to two topology control problems (the Connectivity Game and the Strong Connectivity Game) in static ad hoc networks

\footnotetext{
${ }^{1}$ Roughly speaking, the core is the set of all allocations such that no group of players is "mistreated".
} 
studied by Eidenbenz et al. [7]. The input of these problems is a graph $H=(V, E)$ where each edge $e$ has a weight $w(e) \geq 0$ ( $w$ satisfies the triangle inequality).

In the Connectivity Game [7] we are given pairs $\left(s_{i}, t_{i}\right) \in V \times V$ such that $s_{i}$ needs to connect to $t_{i}$ (possibly over several intermediate vertices). Each $s_{i}$ has to choose a radius. The radius function $\rho$ is a mapping from $V$ to $\mathbb{R}_{+}$ $\left(\rho_{v} \geq 0\right.$ denotes the radius of $v$ ). It induces a directed graph $G_{\rho}=(V, A)$ where $A=\left\{(u, v):[u, v] \in E\right.$ and $\left.\rho_{u} \geq w([u, v])\right\} . G_{\rho}$ is called the transmission graph [12], i.e. $s$ connects to $t$ if there is a directed path from $s$ to $t$ in $G_{\rho}$. The utility of $s_{i}$ is defined as $-M$ if $s_{i}$ does not connect to $t_{i}$ in $G_{\rho}$ ( $M$ being some very large number), otherwise it is $-\left(\rho_{s_{i}}\right)^{\alpha}$. Here $\alpha \geq 1$ is a constant known as the power gradient [12]. So, each $s_{i}$ is a selfish agent whose best strategy is the minimum radius that connects her to $t_{i}$. The social cost is defined as $\sum_{v \in V}\left(\rho_{v}\right)^{\alpha}$. Deciding whether an instance of the Connectivity Game has a pure NE is an NP-complete problem [7]. The Strong Connectivity Game [7] is a special case of the Connectivity Game where each vertex needs to connect with every other vertex. It always has a pure Nash equilibrium and its PoA is $\Theta\left(n^{\alpha}\right)$.

The min SST-game is a particular case of the Connectivity Game where $t_{i}=r$ for all $i=1, \ldots, k,\left\{s_{i}: i=1, \ldots, k\right\}=V \backslash\{r\}$ and $\alpha=1$. It can be also viewed as a relaxation of the Strong Connectivity Game where each vertex needs to connect to $r$ (or if we consider the transmission graph as an unoriented graph, each vertex is connected to every other vertex).

\section{The min sST-game}

Strong-Nash equivalence. A strong equilibrium is a Nash equilibrium but a Nash equilibrium is not necessarily strong (e.g. the prisoner's dilemma). We show that the min SST-game has a particular structure since any Nash equilibrium is strong. This property is known as the strong-Nash equivalence [10].

Theorem 1. Any Nash equilibrium of the min SST-game is a strong equilibrium.

Proof. Given $i \in N$ and a Nash equilibrium $\sigma$, let $D E P(i, \sigma)$ be the players who depend on $i$ to be connected to $r: D E P(i, \sigma)=\left\{j \in N \backslash\{i\}: u_{i}\left(\sigma \mid{ }_{i} j\right)=-\infty\right\}$. Suppose that $\sigma$ is a Nash equilibrium but not a strong equilibrium. Then there exists a coalition $N^{\prime} \subseteq N$ and a strategy profile $\beta$ such that $u_{i}\left(\sigma \mid{ }_{N^{\prime}} \beta\right)>u_{i}(\sigma)$ for all $i \in N^{\prime}$. W.l.o.g., we suppose that $\left(\left.\sigma\right|_{N^{\prime}} \beta\right)=\beta$.

If there exists $i^{\prime} \in N^{\prime}$ such that $\beta_{i^{\prime}} \notin D E P\left(i^{\prime}, \sigma\right)$ then $u_{i^{\prime}}\left(\left.\sigma\right|_{i^{\prime}} \beta_{i^{\prime}}\right)=$ $u_{i^{\prime}}\left(\left.\sigma\right|_{N^{\prime}} \beta\right)>u_{i^{\prime}}(\sigma)$. Since $i^{\prime}$ can unilaterally change her strategy and improve her utility, $\sigma$ is not a Nash equilibrium, contradiction.

If $\beta_{i} \in D E P(i, \sigma)$ for all $i \in N^{\prime}$ then $\left(\left.\sigma\right|_{N^{\prime}} \beta\right)$ does not connect any $i \in N^{\prime}$ to $r$. To see this, let $L^{\prime}=\bigcup_{i \in N^{\prime}} D E P(i, \sigma) \backslash N^{\prime}$ and $U^{\prime}=N \backslash\left(N^{\prime} \cup L^{\prime}\right)$. Actually $L^{\prime}, N^{\prime}$ and $U^{\prime}$ form a partition of $N$. The strategy of a node in $L^{\prime} \cup N^{\prime}$ is to select a node in $L^{\prime} \cup N^{\prime}$. The strategy of a node in $U^{\prime}$ is to select a node in $U^{\prime} \cup\{r\}$. Therefore $u_{i}\left(\left.\sigma\right|_{N^{\prime}} \beta\right)=-\infty<u_{i}(\sigma)$ for all $i \in N^{\prime}$, contradiction.

Then we only consider the price of anarchy of the min SST-game but our results also hold for the strong price of anarchy. 
Definitions and properties. The utility of each player (to be maximized) is the negative of a cost (to be minimized). Given $i \in N$ and $\sigma \in S$, the cost of $i$ is denoted by $c_{i}(\sigma)$ and defined as $-u_{i}(\sigma)$. For the sake of convenience, we often manipulate the cost of a player (which is a non negative value) instead of her utility (which is a non positive value).

We introduce several definitions which will be useful in the next proofs and give two basic properties. In the following, $\sigma$ denotes a strategy profile at Nash equilibrium. Meanwhile $\sigma^{*}$ denotes a social optimum, that is $\sum_{i \in N} c_{i}\left(\sigma^{*}\right)$ is minimum.

Definition 1. Given $(i, j) \in V \times V$, let path ${ }^{*}(i, j)$ be the path (i.e. set of edges) between $i$ and $j$ in $T^{\sigma^{*}}$. Let weight ${ }^{*}(i, j)=\sum_{e \in \text { path }}(i, j)$ w(e) and visited ${ }^{*}(i, j) \subseteq$ $V$ be all nodes of path ${ }^{*}(i, j)$. Let root ${ }^{*}(i, j)$ be the vertex of visited $(i, j)$ which plays the role of the source, i.e. root $^{*}(i, j)=r$ if $r \in \operatorname{visited}^{*}(i, j)$, otherwise $\operatorname{root}^{*}(i, j)=\ell$ where $\ell \in \operatorname{visited}^{*}(i, j)$ and $\sigma_{\ell}^{*} \notin \operatorname{visited}^{*}(i, j)$. Let path, weight, visited and root be defined similarly w.r.t. the tree induced by $\sigma$.

Property 1. If $\sigma$ is a Nash equilibrium then $c_{i}(\sigma) \leq w([i, r])$ holds for all $i \in N$.

Property 2. If $\sigma$ is a Nash equilibrium then $\min \left\{c_{i}(\sigma), c_{j}(\sigma)\right\} \leq w([i, j])$ holds for all $(i, j) \in N \times N$ such that $i \neq j$.

The PoA according to the number of players. We start with a disappointing observation: the players of the min SST-game can arbitrarily deviate from the social optimum. Consider an instance with two players 1 and 2. The weight function is defined as $w([1,2])=w([1, r])=1$ and $w([2, r])=X$ where $X>1$. The strategy profile $\sigma^{*}$ where $\sigma_{1}^{*}=r$ and $\sigma_{2}^{*}=1$ is a social optimum. The strategy profile $\sigma$ where $\sigma_{1}=2$ and $\sigma_{2}=r$ is a Nash equilibrium since 1 has no incentive to change her strategy. Therefore the price of anarchy $\mathcal{S}(\sigma) / \mathcal{S}\left(\sigma^{*}\right)=(1+X) / 2$ tends towards $\infty$ when $X$ tends towards $\infty$. This instance does not satisfy the standard hypothesis which says that the cost of a link depends on its length. From now on, we assume that weights satisfy the triangle inequality, i.e. $w([x, y])+w([y, z]) \geq w([x, z])$ for all triple of nodes $x, y$ and $z$.

Let us give a lower bound on the PoA of the min SST-game.

Proposition 1. When the triangle inequality holds, the PoA of the min SSTgame is at least $1+(\log n) / 2$.

Proof. We consider an instance of the game with $2^{k}$ players for all positive integer $k$. Players are numbered from 1 to $2^{k}$. We assume that the source node $r$ has number 0 . The weight function is defined as $w([i, j])=|i-j|$ for all pair of nodes $(i, j)$. It is not difficult to see that the triangle inequality holds. The minimum weighted tree (an optimal solution), denoted by $T_{k}^{*}$, consists of all edges $[i, i+1]$ $\left(i=0, \ldots, 2^{k}-1\right)$. Its total weight is $2^{k}$.

We are going to describe a spanning tree $T_{k}$ such that the strategy profile induced by $T_{k}$ is a Nash equilibrium for the min SST-game. $T_{k}$ consists of the edges $\left[2^{k-1}, 2^{k}\right],\left[0,2^{k}\right]$ and a subtree $\mathcal{T}_{k}$ which is a complete binary tree on 
vertices $1, \ldots, 2^{k}-1$. To construct $\mathcal{T}_{k}$, we start from a complete binary tree on $2^{k}-1$ vertices which are subsequently numbered.

Each leaf of $\mathcal{T}_{k}$ receives an odd integer between 1 and $2^{k}-1$. The numbering follows the DFS order. Next the number of a non-leaf vertex is defined as the average number of its two children. For instance the root of $\mathcal{T}_{k}$ has number $2^{k-1}$ and it is denoted by $\mathrm{R}_{k}$ in the following. Figure 1 on page 13 gives an illustration of $\mathcal{T}_{2}$ and $\mathcal{T}_{3}$. It suffices to make the union of $\left\{\left[2^{k-1}, 2^{k}\right],\left[0,2^{k}\right]\right\}$ and $\mathcal{T}_{k}$ to get $T_{k}$. Figure 2 on page 13 gives an illustration of $T_{k}^{*}$ and $T_{k}$ when $k=3$.

Given $i \in\{1, \ldots, k\}$, let $N_{i}$ be the set of vertices of $\mathcal{T}_{k}$ whose distance (i.e. number of edges) to $\mathrm{R}_{k}$ in $\mathcal{T}_{k}$ is $i-1$. In particular $N_{1}=\left\{\mathrm{R}_{k}\right\}, N_{k}$ contains all leaves of $\mathcal{T}_{k}$ and $\left|N_{i}\right|=2^{i-1}$. The following properties can be easily shown by induction:

(i) The numbering of the vertices of $N_{i}$ is $\left\{2^{k-i}(2 j-1): j=1, \ldots, 2^{i-1}\right\}$.

(ii) Denote by $p(v)$ the father of $v$ in $\mathcal{T}_{k}$. If $v \in N_{i}$ with $i \in\{2, \ldots, k\}$ then $w([v, p(v)])=2^{k-i}$.

(iii) Given a tree $T$ and a node $v$ of $T$, we denote by $S u b(T, v)$ the subtree of $T$ rooted at $v$. If $v_{0} \in N_{i_{0}}$ with $i_{0} \neq k$ has the number $2^{k-i_{0}}\left(2 j_{0}-1\right)$ for some $j_{0} \in\left\{1, \ldots, 2^{i_{0}-1}\right\}$ then the number of the vertices of $\operatorname{Sub}\left(\mathcal{T}_{k}, v_{0}\right)$ are the integers between $2^{k-i_{0}+1}\left(j_{0}-1\right)+1$ and $2^{k-i_{0}+1} j_{0}-1$, that is $\left\{2^{k-i_{0}+1}\left(j_{0}-\right.\right.$ 1) $\left.+1, \ldots, 2^{k-i_{0}+1} j_{0}-1\right\}$.

Let us prove that the strategy profile induced by $T_{k}$ is a Nash equilibrium. By construction a vertex $v$ of $\mathcal{T}_{k}$ can be linked to any vertex except those of $\operatorname{Sub}\left(\mathcal{T}_{k}, v\right)$ (otherwise $v$ is not connected to $r$ anymore). So, assume that $v \in N_{i_{0}}$ has number $2^{k-i_{0}}\left(2 j_{0}-1\right)$ for some $i_{0} \in\{1, \ldots, k\}$ and $j_{0} \in\left\{1, \ldots, 2^{i_{0}-1}\right\}$. By Property $(\mathrm{iii})$, we know that the two vertices $v_{0}$ and $v_{1}$ closest to $v$ are numbered $2^{k-i_{0}+1}\left(j_{0}-1\right)$ and $2^{k-i_{0}+1} j_{0}$ respectively. Thus, we deduce that $w\left(\left[v, v_{0}\right]\right)=$ $\left|2^{k-i_{0}}\left(2 j_{0}-1\right)-2^{k-i_{0}+1}\left(j_{0}-1\right)\right|=2^{k-i_{0}}$ and $w\left(\left[v, v_{1}\right]\right)=\mid 2^{k-i_{0}}\left(2 j_{0}-1\right)-$ $2^{k-i_{0}+1} j_{0} \mid=2^{k-i_{0}}$. By Property $(i i)$, these distances are equals to $w([v, p(v)])$. Finally, by observing that the distance of $\mathrm{R}_{k}$ (numbered $2^{k-1}$ ) to $r$ is equal to the distance of $\mathrm{R}_{k}$ to $2^{k}$, we conclude that $T_{k}$ is a Nash equilibrium. Now, using Properties $(i)$ and $(i i)$, we deduce that the total weight of $T_{k}$ is $2^{k}+2^{k-1}+$ $\sum_{i=1}^{k}\left|N_{i}\right| 2^{k-i}=2^{k}+2^{k-1}+(k-1) 2^{k-1}=(k+2) 2^{k-1}$. Then the price of anarchy of the min SST-game is at least $\left((k+2) 2^{k-1}\right) / 2^{k}=(k+2) / 2=1+\frac{\log n}{2}$ since $n=2^{k}$.

Before giving an upper bound on the PoA of the min ssT-game we prove an intermediate result which is useful in the subsequent proof.

Lemma 1. Given an oriented tree $T=(V, E)$ rooted at $r$ on $n+1$ vertices, and for any subset $V^{\prime}=\left\{v_{1}, \ldots, v_{2 p+1}\right\} \subseteq V \backslash\{r\}$ of $2 p+1$ vertices (hence, $2 p+1 \leq n)$, one can exhibit $p+1$ edge disjoint unoriented paths path for $i=1, \ldots, p+1$ in $T$ such that one path, say path $h_{p+1}$, is a path from $v_{i}$ to $r$ for $i \leq 2 p+1$ whereas the endpoints set of the $p$ other paths is exactly $V^{\prime} \backslash\left\{v_{i}\right\}$.

Let us return to the PoA of the min sST-game. 
Theorem 2. When the triangle inequality holds, the PoA of the min SST-game is at most $\lfloor\log n\rfloor+1+\frac{2}{n}$.

Proof. Let $\sigma$ be a Nash equilibrium while $\sigma^{*}$ denotes a social optimum. We suppose that the players are sorted by decreasing cost, i.e. $c_{1}(\sigma) \geq \cdots \geq c_{n}(\sigma)$ where $n=|N|$. In order to keep simple, we assume that $n$ is odd. We mainly prove the following property:

$$
\forall p=0, \ldots, \frac{n-1}{2}, \quad \sum_{i=p+1}^{2 p+1} c_{i}(\sigma) \leq \sum_{i=1}^{n} c_{i}\left(\sigma^{*}\right)
$$

Suppose that (1) holds and replace $p$ by $2^{j}-1$. One has $\sum_{i=2^{j}}^{2^{j+1}-1} c_{i}(\sigma) \leq$ $\sum_{i=1}^{n} c_{i}\left(\sigma^{*}\right)$ and the result follows:

$$
\sum_{i=1}^{n} c_{i}(\sigma) \leq \sum_{j=0}^{\lfloor\log n\rfloor-1} \sum_{i=2^{j}}^{2^{j+1}-1} c_{i}(\sigma)+\sum_{i=\frac{n+1}{2}}^{n} c_{i}(\sigma) \leq(\lfloor\log n\rfloor+1) \sum_{i=1}^{n} c_{i}\left(\sigma^{*}\right)
$$

If $n$ is even then we conduct the same proof on the $n-1$ first players and we use the fact that $\frac{n}{2} c_{n}(\sigma) \leq \sum_{i=\frac{n}{2}}^{n-1} c_{i}(\sigma) \leq \sum_{i=1}^{n} c_{i}\left(\sigma^{*}\right)$. Then it suffices to prove inequality (1).

Given $p \in\left\{0, \ldots, \frac{n-1}{2}\right\}$, let $V_{p}$ be the $2 p+1$ players with largest costs, i.e. $V_{p}=\{1, \ldots, 2 p+1\}$. Let $T^{*}$ be the tree induced by $\sigma^{*}$. The total weight of $T^{*}$ is denoted by $O P T$; it is equal to $\sum_{i=1}^{n} c_{i}\left(\sigma^{*}\right)$. We know from Lemma 1 with input $V_{p}$ that one can find $p+1$ edge-disjoint paths in $T^{*}$. W.l.o.g., assume that these paths are $\operatorname{path}^{*}(\pi(2 i-1), \pi(2 i))$ for $i=1, \ldots, p$ and $\operatorname{path}^{*}(\pi(2 p+1), r)$ where $\pi$ is a permutation of $V_{p}$. We deduce:

$$
\sum_{i=1}^{p+1} \operatorname{weight}^{*}(\pi(2 i-1), \pi(2 i)) \leq O P T .
$$

By the triangle inequality, we also get:

$$
w([\pi(2 i-1), \pi(2 i)]) \leq \text { weight }^{*}(\pi(2 i-1), \pi(2 i))
$$

for $i=1, \ldots, p$ and

$$
w([\pi(2 p+1), r]) \leq \operatorname{weight}^{*}(\pi(2 p+1), r) .
$$

Using Property 2 we have:

$$
\min \left\{c_{\pi(2 i-1)}(\sigma), c_{\pi(2 i)}(\sigma)\right\} \leq w([\pi(2 i-1), \pi(2 i)]) \text { for } i=1, \ldots, p
$$

Using Property 1 , the cost of player $\pi(2 p+1)$ is less than $w([\pi(2 p+1), r])$ if she is connected to $r$ via another player in $\sigma$. Therefore

$$
c_{\pi(2 p+1)}(\sigma) \leq w([\pi(2 p+1), r])
$$


Since players are numbered by decreasing cost, we have

$$
\sum_{i=p+1}^{2 p+1} c_{i}(\sigma) \leq \sum_{i=1}^{p} \min \left\{c_{\pi(2 i-1)}(\sigma), c_{\pi(2 i)}(\sigma)\right\}+c_{\pi(2 p+1)}(\sigma) .
$$

Summing up inequalities (5) for $i=1, \ldots, p$ and adding inequality (6) we get:

$$
\begin{aligned}
\sum_{i=1}^{p} \min \left\{c_{\pi(2 i-1)}(\sigma), c_{\pi(2 i)}(\sigma)\right\}+c_{\pi(2 p+1)}(\sigma) \leq & \sum_{i=1}^{p} w([\pi(2 i-1), \pi(2 i)]) \\
& +w([\pi(2 p+1), r])
\end{aligned}
$$

Finally, using inequalities (7), (8), (2),(3) and (4), we obtain inequality (1), that is $\sum_{i=p+1}^{2 p+1} c_{i}(\sigma) \leq O P T$.

Therefore the PoA of the min SST-game belongs to $\Theta(\log n)$. One can generalize the min SST-game to the case where the social cost is defined as $\sum_{i=1}^{n}\left(c_{i}(\sigma)\right)^{\alpha}$ (problem denoted by min SST-game $(\alpha)$ in the following). Here $\alpha$ is a constant greater than 1 such that $d^{\alpha}$ is the minimum power to transmit a message at distance $d[12]$.

Theorem 3. For any $\alpha \geq 1$, the PoA of the min SST-game $(\alpha)$ is upper bounded by $n^{\alpha-1}\left(\lfloor\log n\rfloor+1+\frac{2}{n}\right)$.

The PoA according to the depth of an optimum tree. We propose an alternative bound on the PoA of the min SST-game. We need some notations and two intermediate results to show the result.

Let $\mathcal{T}$ be an oriented tree rooted at $r$ in the following definitions. Given $i \in N$, let $\mathcal{T}_{i}$ be the subtree of $\mathcal{T}$ rooted at $i$. Let $V(\mathcal{T})$ be the set of vertices spanned by $\mathcal{T}$. The weight of $\mathcal{T}$, denoted by $w(\mathcal{T})$, is defined as $\sum_{e \in \mathcal{T}} w(e)$. The depth of $\mathcal{T}$, denoted by $\operatorname{depth}(\mathcal{T})$, is defined as $\max _{i \in N}\left\{\left|\operatorname{path}_{\mathcal{T}}(i, r)\right|\right\}$ where $\operatorname{path}_{\mathcal{T}}(i, r)$ is the path (i.e. set of edges) between $i$ and $r$ in $\mathcal{T}$. The level of $i \in N$ in $\mathcal{T}$, denoted by $\ell(\mathcal{T}, i)$, is defined as $\mid$ path $_{\mathcal{T}}(i, r) \mid$. Given $N^{\prime} \subseteq N$, let $f\left(\mathcal{T}, N^{\prime}\right)$ be a node of $N^{\prime}$ with minimum level in $\mathcal{T}$, i.e. $f\left(\mathcal{T}, N^{\prime}\right)=\operatorname{argmin}_{i \in N^{\prime}}\{\ell(\mathcal{T}, i)\}$.

Lemma 2. We are given a spanning tree $\mathcal{T}$ of $G=(V, E)$ rooted at $r$, a spanning tree $T$ induced by a Nash equilibrium $\sigma$, a player $i \in N$ and $H \subseteq V\left(\mathcal{T}_{i}\right) \backslash\{i\}$ such that $\forall a, b \in H, a \notin \mathcal{T}_{b}$ and $b \notin \mathcal{T}_{a}$. Let $i^{*}$ be the element of $H \cup\{i\}$ with minimum level in $T$, i.e. $i^{*}=f(T, H \cup\{i\})$. If the triangle inequality holds then

$$
\left(\sum_{j \in H \cup\{i\}} c_{j}(\sigma)\right)-c_{i^{*}}(\sigma) \leq 2\left(\sum_{j \in H} w([i, j])\right)-w\left(\left[i, i^{*}\right]\right) .
$$

Proposition 2. We are given a spanning tree $\mathcal{T}$ of $G=(V, E)$ rooted at $r$, a spanning tree $T$ induced by a Nash equilibrium $\sigma$ and $i \in N$ (i.e., $i \neq r$ ). Let $i^{*}=f\left(T, V\left(\mathcal{T}_{i}\right)\right)$. If the triangle inequality holds then

$$
\sum_{j \in V\left(\mathcal{T}_{i}\right)} c_{j}(\sigma)-c_{i^{*}}(\sigma) \leq\left(\operatorname{depth}\left(\mathcal{T}_{i}\right)+1\right) w\left(\mathcal{T}_{i}\right)-w\left(\left[i, i^{*}\right]\right) .
$$


Now we are able to prove that the PoA is upper bounded by the depth of a minimum weight spanning tree rooted at $r$ (denoted by $d^{*}$ in the following). For instance the case $d^{*}=1$ is a direct consequence of Property 1 .

Theorem 4. When the triangle inequality holds, the PoA of the min SST-game is at most $d^{*}$ where $d^{*}$ is the depth of a minimum weight spanning tree rooted at $r$.

Proof. Let $T^{*}$ be a minimum weight spanning tree rooted at $r$ with depth $d^{*}$. $T^{*}$ is induced by a social optimum $\sigma^{*}$. Let $T$ be the tree induced by a Nash equilibrium $\sigma$. Denote by $1, \ldots, k$ the sons of $r$ in $T^{*}$, i.e. $\left\{i \in N:[i, r] \in T^{*}\right\}=$ $\{1, \ldots, k\}$. Proposition 2 for any $i \in\{1, \ldots, k\}$ gives

$$
\sum_{j \in V\left(T_{i}^{*}\right)} c_{j}(\sigma) \leq\left(\operatorname{depth}\left(T_{i}^{*}\right)+1\right) w\left(\mathcal{T}_{i}^{*}\right)-w\left(\left[i, i^{*}\right]\right)+c_{i^{*}}(\sigma)
$$

where $i^{*}=f\left(T, V\left(T_{i}^{*}\right)\right)$. Using Property 1 and the triangle inequality we obtain $c_{i^{*}}(\sigma) \leq w\left(\left[r, i^{*}\right]\right) \leq w([r, i])+w\left(\left[i, i^{*}\right]\right)$. Then inequality (9) becomes:

$$
\sum_{j \in V\left(T_{i}^{*}\right)} c_{j}(\sigma) \leq\left(\operatorname{depth}\left(T_{i}^{*}\right)+1\right)\left(w\left(T_{i}^{*}\right)+w([r, i])\right)
$$

Using $\operatorname{depth}\left(T_{i}^{*}\right)+1 \leq \operatorname{depth}\left(T^{*}\right)$ and summing up inequality $(10)$ for $j=1, \ldots, k$ we get:

$$
\begin{aligned}
& \sum_{i=1}^{k} \sum_{j \in V\left(T_{i}^{*}\right)} c_{j}(\sigma) \leq \operatorname{depth}\left(T^{*}\right) \sum_{j=1}^{k}\left(w\left(T_{i}^{*}\right)+w([r, i])\right) \\
& \sum_{j \in N} c_{j}(\sigma) \leq \operatorname{depth}\left(T^{*}\right) w\left(T^{*}\right)=\operatorname{depth}\left(T^{*}\right) \sum_{j \in N} c_{j}\left(\sigma^{*}\right) .
\end{aligned}
$$

\section{The max SST-game and the bottleneck SST-game}

In contrast with the previous section we do not suppose that the triangle inequality holds. Let us begin with the max SST-game. It is noteworthy that the proof of Theorem 1 also works for the max SST-game. Then any Nash equilibrium of the max SST-game is strong. Again we only study the PoA since PoA=SPoA.

Theorem 5. The PoA the max SST-game is $1 / \Delta^{*}$ where $\Delta^{*}$ is the maximum degree of a player node in a maximum cost spanning tree.

Proof. We know from that $w([i, j]) \leq \max \left\{u_{i}(\sigma), u_{j}(\sigma)\right\}$ for any pair of players $(i, j)$ (the property is similar to Property 2). Moreover $w([i, r]) \leq u_{i}(\sigma)$ holds for all $i \in N$ (the property is similar to Property 1). We use these inequalities for each edge of the optimal tree as follows.

$$
\begin{aligned}
& \mathcal{S}\left(\sigma^{*}\right):=\sum_{\left\{i \in N: \sigma_{i}^{*} \neq r\right\}} w\left(\left[i, \sigma_{i}^{*}\right]\right)+\sum_{\left\{i \in N: \sigma_{i}^{*}=r\right\}} w([i, r]) \\
& \mathcal{S}\left(\sigma^{*}\right) \leq \sum_{\left\{i \in N: \sigma_{i}^{*} \neq r\right\}} \max \left\{u_{i}(\sigma), u_{\sigma_{i}^{*}}(\sigma)\right\}+\sum_{\left\{i \in N: \sigma_{i}^{*}=r\right\}} u_{i}(\sigma)
\end{aligned}
$$


A player's utility may appear several times in the upper bound but at most $\Delta^{*}$ times. Hence, $\mathcal{S}\left(\sigma^{*}\right) \leq \Delta^{*} \sum_{i \in N} u_{i}(\sigma)=\Delta^{*} \mathcal{S}(\sigma)$ and $\operatorname{PoA}=\mathcal{S}(\sigma) / \mathcal{S}\left(\sigma^{*}\right) \geq$ $1 / \Delta^{*}$. Tight examples (with and without the triangle inequality) are given in the appendix.

Theorem 6. The PoA of the bottleneck SST-game is 1 .

Proof. Let $\sigma^{*}$ be a strategy profile which maximizes the social welfare. Let $\sigma$ be a Nash equilibrium. We denote by $T^{*}$ (resp., $T$ ) the tree induced by $\sigma^{*}$ (resp., $\sigma)$. Let $B=\left\{i \in N: u_{i}(\sigma)<u_{i}\left(\sigma^{*}\right)\right\}$ be the players whose utility is suboptimal. Denote by $h(i)$ the number of edges which compose the unique path between node $i$ and the source $r$ along $T^{*}$. Let $i^{*}=\operatorname{argmin}\{h(i): i \in B\}$ (ties are arbitrarily broken).

If $h\left(i^{*}\right)=1$ then $u_{i^{*}}\left(\sigma \mid i_{i^{*}} r\right)=u_{i^{*}}\left(\sigma^{*}\right)$. However $u_{i^{*}}(\sigma) \geq u_{i^{*}}\left(\left.\sigma\right|_{i^{*}} r\right)$ always holds because $\sigma$ is a Nash equilibrium. We get $u_{i^{*}}(\sigma) \geq u_{i^{*}}\left(\sigma^{*}\right)$ which is in contradiction with $i^{*} \in B$. Therefore $h\left(i^{*}\right)>1$. Let $j^{*} \in N$ be such that $\sigma_{i^{*}}^{*}=j^{*}$. By definition, we have

$$
u_{i^{*}}\left(\sigma^{*}\right)=\min \left\{u_{j^{*}}\left(\sigma^{*}\right), w\left(\left[i^{*}, j^{*}\right]\right)\right\}
$$

We know that $j^{*} \notin B$ because $h\left(j^{*}\right)<h\left(i^{*}\right)$. We deduce

$$
u_{j^{*}}\left(\sigma^{*}\right) \leq u_{j^{*}}(\sigma)
$$

Moreover $u_{i^{*}}\left(\left.\sigma\right|_{i^{*}} j^{*}\right)=-\infty$ since by (11) and (12) we have

$$
\left.u_{i^{*}}\left(\sigma^{*}\right) \leq \min \left\{u_{j^{*}}(\sigma), w\left(\left[i^{*}, j^{*}\right]\right)\right\}=u_{i^{*}}\left(\left.\sigma\right|_{i^{*}}, j^{*}\right)\right) .
$$

Now, since $i^{*} \in B$, we deduce $\left.u_{i^{*}}\left(\left.\sigma\right|_{i^{*}} j^{*}\right)\right) \geq u_{i^{*}}\left(\sigma^{*}\right)>u_{i^{*}}(\sigma)$ would contradict the fact that $\sigma$ is a Nash equilibrium. Then $i^{*} \in \operatorname{visited}\left(j^{*}, r\right)$ implies

$$
u_{j^{*}}(\sigma) \leq u_{i^{*}}(\sigma)
$$

Using inequalities (11), (12) and (13), we get $u_{i^{*}}\left(\sigma^{*}\right) \leq u_{i^{*}}(\sigma)$ which is in contradiction with $i^{*} \in B$. Then $B$ must be empty and $u_{i}(\sigma) \geq u_{i}\left(\sigma^{*}\right)$ holds for all $i \in N$. The result follows since $\mathcal{S}\left(\sigma^{*}\right) \geq \mathcal{S}(\sigma)=\sum_{i \in N} u_{i}(\sigma) \geq \sum_{i \in N} u_{i}\left(\sigma^{*}\right)=$ $\mathcal{S}\left(\sigma^{*}\right)$.

Because a strong equilibrium is a Nash equilibrium, $\mathrm{PoA} \leq \mathrm{SPoA} \leq 1$ holds for the bottleneck SST-game. Then the SPoA of the bottleneck SST-game is 1 and any Nash equilibrium is a strong equilibrium.

\section{Concluding remarks}

We prove that a Nash equilibrium is a strong equilibrium for all games under consideration. Then, is cooperation inefficient or inherent? Actually the answer is probably that cooperation is inefficient. On the one hand cooperating with a player who depends on you to be connected (i.e. you are on her path to $r$ ) can be 
profitable. On the other hand cooperating with a player who do not depend on you to be connected (i.e. you are not on her path to $r$ ) is never profitable. Since two players cannot mutually depend on the other to connect to $r$, cooperation is unlikely.

We study three variants of a strategic spanning tree game but the focus is on the min SST-game. The gap between the lower bound $(1+(\log n) / 2)$ and the upper bound $(\lfloor\log n\rfloor+1+n / 2)$ is quite narrow but it is certainly possible to give a better analysis of the upper bound.

The same goes for Theorem 3 (when the social cost of the min SST-game is $\left.\sum_{i \in N}\left(c_{i}(\sigma)\right)^{\alpha}\right)$, we believe that a more accurate upper bound can be derived.

We were not able to find tight examples for Theorem 4 except when $d^{*}=2$ (see Proposition 3 in the appendix). Nevertheless we are not convinced that such instances exist for $d^{*}>2$. Then it would be interesting to improve the upper bound $d^{*}$.

\section{References}

1. S. Albers, On the value of coordination in network design, In Proc. of SODA 2008, pp. 294-303, 2008.

2. N. Andelman, M. Feldman and Y. Mansour, Strong price of anarchy, In Proc. of SODA 2007, pp. 189-198, 2007.

3. E. Anshelevich, A. Dasgupta, J. M. Kleinberg, É. Tardos, T. Wexler and T. Roughgarden. The Price of Stability for Network Design with Fair Cost Allocation, In Proc. of FOCS 2004, pp. 295-304, 2004.

4. R. Aumann, Acceptable points in general cooperative n-person games, In Contribution to the Theory of Games, vol. IV, Annals of Mathematics Studies, 40, pp. 287-324, 1959.

5. C.G. Bird, On cost allocation for a spanning tree: A game theory approach, in Networks, volume 6, pp. 335-350, 1976.

6. A. Claus and D. Kleitman, Cost allocation for a spanning tree, Networks, vol 3, pp 289-304,1973.

7. S. Eidenbenz, V. S. A. Kumar and S. Zust, Equilibria in topology control games for ad hoc networks, in Proc. of DIALM-POMC, pp. 2-11, 2003.

8. D. Granot and G. Huberman, Minimum cost spanning tree games, Mathematical Programming, vol 21, pp 1-18, 1981.

9. D. Granot and G. Huberman, On the core and nucleolus of minimum cost spanning tree games, Mathematical Programming, vol 29, pp 323-347, 1984.

10. R. Holzman and N. Law-Yone, Strong Equilibrium in Congestion Games, Games and economic behavior, vol 21, pp 85-101, 1997.

11. E. Koutsoupias and C. H. Papadimitriou, Worst Case Equilibria. In Proc. of STACS 1999, LNCS 1563, pp. 404-413, 1999.

12. R. Rajaraman, Topology control and routing in ad hoc networks: a survey, In SIGACT News, 33, pp. 60-73, 2002. 


\section{Appendix}

Property 1. If $\sigma$ is a Nash equilibrium then $c_{i}(\sigma) \leq w([i, r])$ holds for all $i \in N$.

Proof. Whatever the other players do in $\sigma$, the cost of player $i$ is $w([i, r])$ if her strategy is to select her direct link to $r$. If $i$ is connected to $r$ via an intermediate node at a cost strictly larger than $w([i, r])$ then $\sigma$ is not a Nash equilibrium.

Property 2 If $\sigma$ is a Nash equilibrium then $\min \left\{c_{i}(\sigma), c_{j}(\sigma)\right\} \leq w([i, j])$ holds for all $(i, j) \in N \times N$ such that $i \neq j$.

Proof. If $i \notin \operatorname{visited}(j, r)$ then $\min \left\{c_{i}(\sigma), c_{j}(\sigma)\right\} \leq c_{i}(\sigma) \leq c_{i}\left(\left.\sigma\right|_{i} j\right)=w([i, j])$. Similarly, if $j \notin \operatorname{visited}(i, r)$ then $\min \left\{c_{i}(\sigma), c_{j}(\sigma)\right\} \leq c_{j}(\sigma) \leq c_{j}\left(\left.\sigma\right|_{j} i\right)=$ $w([i, j])$. Finally, $i \in \operatorname{visited}(j, r)$ and $j \in \operatorname{visited}(i, r)$ cannot simultaneously hold because the graph induced by $\sigma$ must be a tree.

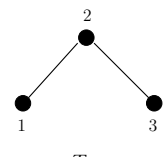

$T_{2}$

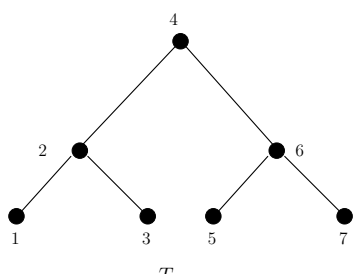

$T_{3}$

Fig. 1. Numbering of $\mathcal{T}_{2}$ and $\mathcal{T}_{3}$.

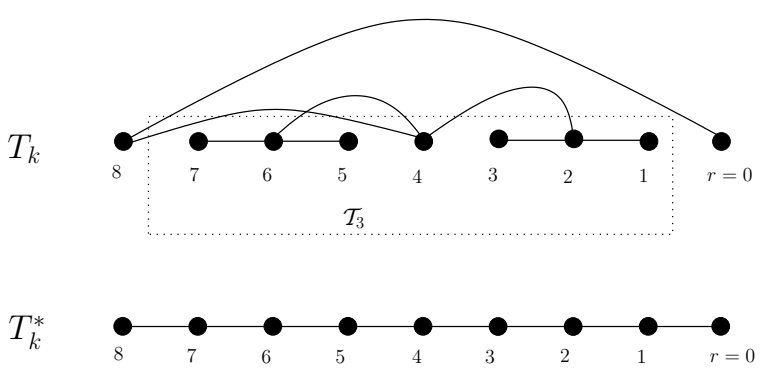

Fig. 2. $T_{k}$ and $T_{k}^{*}$ when $k=3$.

Lemma 1. Given an oriented tree $T=(V, E)$ rooted at $r$ on $n+1$ vertices, and for any subset $V^{\prime}=\left\{v_{1}, \ldots, v_{2 p+1}\right\} \subseteq V \backslash\{r\}$ of $2 p+1$ vertices (hence, $2 p+1 \leq n$ ), one can exhibit $p+1$ edge disjoint unoriented paths path ${ }_{i}$ for $i=1, \ldots, p+1$ in $T$ such that one path, say path ${ }_{p+1}$, is a path from $v_{i}$ to $r$ for $i \leq 2 p+1$ whereas the endpoints set of the $p$ other paths is exactly $V^{\prime} \backslash\left\{v_{i}\right\}$. 

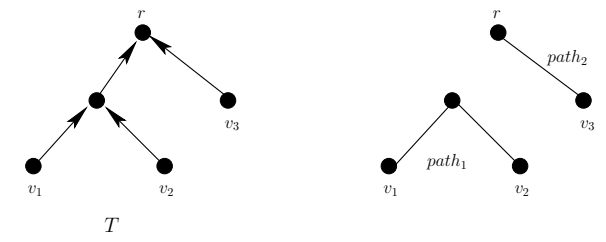

Fig. 3. The construction of path $_{1}$ and path $h_{2}$ when $V^{\prime}=\left\{v_{1}, v_{2}, v_{3}\right\}$.

Proof. We make the proof by induction. For $p=0$, the result is clearly true since path $\left(v_{1}, r\right)$ - the path in $T$ from $v_{1}$ to $r$ - is the solution. Now, assume that this property holds for any oriented tree and for any $\left|V^{\prime}\right|=2 q+1$ with $q<p$ and let us prove that the result holds for $\left|V^{\prime}\right|=2 p+1$.

Let $T=(V, E)$ be an oriented tree rooted at $r$ on $n+1$ vertices, and let $V^{\prime}=\left\{v_{1}, \ldots, v_{2 p+1}\right\} \subseteq V \backslash\{r\}$. For any (unoriented) path $\operatorname{path}\left(v_{i}, v_{j}\right)$ from $v_{i}$ to $v_{j}$ in $T$ where $1 \leq i<j \leq 2 p+1$, let $l(i, j)$ be the level of vertex $\operatorname{root}\left(v_{i}, v_{j}\right)$ (i.e., the length of the path from $\operatorname{root}\left(v_{i}, v_{j}\right)$ to $r$ ) where $\operatorname{root}\left(v_{i}, v_{j}\right)$ is the vertex in $\operatorname{path}\left(v_{i}, v_{j}\right)$ such that the father of $\operatorname{root}\left(v_{i}, v_{j}\right)$ does not belong to $\operatorname{path}\left(v_{i}, v_{j}\right)$.

We choose $i^{*}, j^{*}$ such that $l\left(i^{*}, j^{*}\right)=\max \{l(i, j): 1 \leq i<j \leq 2 p+1\}$ and denote by $T_{v}$ the subtree of $T$ rooted at $v$. Consider the two following cases:

- If $v_{i^{*}} \neq \operatorname{root}\left(v_{i^{*}}, v_{j^{*}}\right)$ and $v_{j^{*}} \neq \operatorname{root}\left(v_{i^{*}}, v_{j^{*}}\right)$. Let $c_{1}$ and $c_{2}$ be the two children of $\operatorname{root}\left(v_{i^{*}}, v_{j^{*}}\right)$ in path $\left(v_{i^{*}}, v_{j^{*}}\right)$. We set $T^{\prime}=T \backslash\left(T_{c_{1}} \cup T_{c_{2}}\right)$ and $V^{\prime \prime}=V^{\prime} \backslash\left\{v_{i^{*}}, v_{j^{*}}\right\}$. Let us verify that $T^{\prime}$ and $V^{\prime \prime}$ satisfy the inductive hypothesis. $T^{\prime}$ is obviously a tree rooted at $r$. Thus, we only proved that $\left(V\left(T_{c_{1}}\right) \cup V\left(T_{c_{2}}\right)\right) \cap V "=\emptyset$. Otherwise, for instance there exists $v_{k} \in V\left(T_{c_{2}}\right) \cap$ $V^{\prime}$. Then, we obtain $\max \left\{l\left(i^{*}, k\right), l\left(j^{*}, k\right)\right\}>l\left(i^{*}, j^{*}\right)$, contradiction.

- If $v_{i^{*}}=\operatorname{root}\left(v_{i^{*}}, v_{j^{*}}\right)$ or $v_{j^{*}}=\operatorname{root}\left(v_{i^{*}}, v_{j^{*}}\right)$. Let us assume $v_{i^{*}}=\operatorname{root}\left(v_{i^{*}}, v_{j^{*}}\right)$. Let $c_{1}$ be the child of $v_{i^{*}}$ in $\operatorname{path}\left(v_{i^{*}}, v_{j^{*}}\right)$. We set $T^{\prime}=T \backslash T_{c_{1}}$ and $V^{\prime \prime}=$ $V^{\prime} \backslash\left\{v_{i^{*}}, v_{j^{*}}\right\}$. Like in the previous case, it is not difficult to verify that $T^{\prime}$ and $V^{\prime \prime}$ satisfy the inductive hypothesis.

In conclusion, using the inductive hypothesis on $T^{\prime}$ and $V^{\prime \prime}$, one can exhibit $p$ edge disjoints paths in $T^{\prime}$ satisfying the condition. These $p$ paths plus $\operatorname{path}\left(v_{i^{*}}, v_{j^{*}}\right)$ form the expected solution. Figure 3 gives an illustration of the above construction for $p=1$.

Theorem 3. For any $\alpha \geq 1$, the PoA of the min SsT-game $(\alpha)$ is upper bounded by $n^{\alpha-1}\left(\lfloor\log n\rfloor+1+\frac{2}{n}\right)$.

Proof. In order to keep simple, we assume that $n$ is odd. Let $\sigma$ be a Nash equilibrium and let $\sigma^{*}$ be the strategy profile induced by an optimal solution $T^{*}$. Let $N A S H(\alpha)=\sum_{i=1}^{n}\left(c_{i}(\sigma)\right)^{\alpha}$ and $O P T(\alpha)=\sum_{e \in T^{*}}(w(e))^{\alpha}=\sum_{i=1}^{n}\left(c_{i}\left(\sigma^{*}\right)\right)^{\alpha}$. Since $x^{\alpha}$ is a nondecreasing function when $x$ is a positive real, we have:

$$
\min \left\{\left(c_{i}(\sigma)\right)^{\alpha},\left(c_{j}(\sigma)\right)^{\alpha}\right\} \leq(w([i, j]))^{\alpha} \text { for all }(i, j) \in N \times N \text { s.t. } i \neq j
$$


Now, assume that the players are sorted by decreasing cost: $c_{1}(\sigma) \geq c_{2}(\sigma) \geq$ $\cdots \geq c_{n}(\sigma)$ where $n=|N|$. Using inequality (14), inequality (1) becomes:

$$
\forall p \in\left\{0, \ldots, \frac{n-1}{2}\right\}, \quad \sum_{i=p+1}^{2 p+1}\left(c_{i}(\sigma)\right)^{\alpha} \leq \sum_{i=1}^{p+1}\left(\text { weight }^{*}(\pi(2 i-1), \pi(2 i))\right)^{\alpha} .
$$

where weight $t^{*}(a, b)$ is the weight of $\operatorname{path}^{*}(a, b)$ - the path between $a$ and $b$ along $T^{*}$ - and $\pi$ is a permutation such that the paths of $\left\{\right.$ path $^{*}(\pi(2 i-1), \pi(2 i))$ : $i=1, \ldots, p\} \cup\left\{\right.$ path $\left.^{*}(\pi(2 p+1), r)\right\}$ are pairwise edge-disjoint. For the ease of presentation let path ${ }_{i}^{*}=\operatorname{path}^{*}(\pi(2 i-1), \pi(2 i))$ if $i \in\{1, \ldots, p\}$ and path $_{p+1}^{*}=$ path $^{*}(\pi(2 p+1), r)$. Therefore weight $^{*}(\pi(2 i-1), \pi(2 i))=\sum_{e \in \text { path }_{i}^{*}} w(e)$ for $i=1, \ldots, p+1$ and (15) becomes

$$
\forall p \in\left\{0, \ldots, \frac{n-1}{2}\right\}, \quad \sum_{i=p+1}^{2 p+1}\left(c_{i}(\sigma)\right)^{\alpha} \leq \sum_{i=1}^{p+1}\left(\sum_{e \in p a t h_{i}^{*}}(w(e))^{\alpha} .\right.
$$

Since $x^{\alpha}$ is a convex function when $x$ is a positive reals $(\alpha \geq 1)$, we deduce:

$$
\forall i \in\{1, \ldots, p+1\}, \quad\left(\sum_{e \in p a t h_{i}^{*}} w(e)\right)^{\alpha} \leq\left|p a t h_{i}^{*}\right|^{\alpha-1} \sum_{e \in p a t h_{i}^{*}}(w(e))^{\alpha}
$$

Summing up inequalities (17) for $i=1, \ldots, p+1$ and using the fact that $\left|p a t h_{i}^{*}\right| \leq$ $n$ we get:

$$
\sum_{i=1}^{p+1}\left(\sum_{e \in \text { path }} w(e)\right)^{\alpha} \leq n^{\alpha-1} \sum_{i=1}^{p+1} \sum_{e \in p a t h_{i}^{*}}(w(e))^{\alpha} \leq n^{\alpha-1} O P T(\alpha)
$$

Using inequalities (16) and (18) we get:

$$
\forall p \in\left\{0, \ldots, \frac{n-1}{2}\right\}, \quad \sum_{i=p+1}^{2 p+1}\left(c_{i}(\sigma)\right)^{\alpha} \leq n^{\alpha-1} \operatorname{OPT}(\alpha)
$$

Replace $p$ by $2^{j}-1$ like in the proof of Theorem 2 and the result follows:

$$
\begin{aligned}
N A S H(\alpha) & \leq \sum_{j=0}^{\lfloor\log n\rfloor-1} \sum_{i=2^{j}}^{2^{j+1}-1}\left(c_{i}(\sigma)\right)^{\alpha}+\sum_{i=\frac{n+1}{2}}^{n}\left(c_{i}(\sigma)\right)^{\alpha} \\
& \leq n^{\alpha-1} \sum_{j=0}^{\lfloor\log n\rfloor} O P T(\alpha)=n^{\alpha-1}(\lfloor\log n\rfloor+1) O P T(\alpha)
\end{aligned}
$$


Lemma 2. We are given a spanning tree $\mathcal{T}$ of $G=(V, E)$ rooted at $r$, a spanning tree $T$ induced by a Nash equilibrium $\sigma$, a player $i \in N$ and $H \subseteq V\left(\mathcal{T}_{i}\right) \backslash\{i\}$ such that $\forall a, b \in H, a \notin \mathcal{T}_{a}$ and $b \notin \mathcal{T}_{b}$. Let $i^{*}$ be the element of $H \cup\{i\}$ with minimum level in $T$, i.e. $i^{*}=f(T, H \cup\{i\})$. If the triangle inequality holds then

$$
\left(\sum_{j \in H \cup\{i\}} c_{j}(\sigma)\right)-c_{i^{*}}(\sigma) \leq 2\left(\sum_{j \in H} w([i, j])\right)-w\left(\left[i, i^{*}\right]\right) .
$$

Proof. Let $k$ be the cardinality of $H$. We use a function $\pi:\{0, \ldots, k\} \mapsto H \cup\{i\}$ such that $\pi(0)=i^{*}$ and $\ell(\mathcal{T}, \pi(a)) \leq \ell(\mathcal{T}, \pi(b))$ iff $a \leq b$. Actually $\pi$ sorts the elements of $H \cup\{i\}$ by increasing level in $T$. Since $T$ is induced by $\sigma, \sigma$ connects to the source any player $\pi(j$ ) (for $1 \leq j \leq k$ ) whose strategy belongs to $\{\pi(0), \ldots, \pi(j-1)\}$. In particular the cost of $\pi(j)$ is $w([\pi(j), \pi(j-1)])$ if her strategy is $\pi(j-1)$. Therefore $c_{\pi(j)}(\sigma)$ is at most $w([\pi(j), \pi(j-1)])$. Furthermore $w([\pi(j), \pi(j-1)])$ is bounded by $w([\pi(j), i])+w([i, \pi(j-1)])$ by the triangle inequality.

$$
\begin{aligned}
\sum_{j=1}^{k} c_{\pi(j)}(\sigma) & \leq \sum_{j=1}^{k} w([\pi(j), \pi(j-1)]) \\
& \leq \sum_{j=1}^{k}(w([\pi(j), i])+w([i, \pi(j-1)])) \\
& \leq 2\left(\sum_{j=1}^{k} w([\pi(j), i])\right)-w\left(\left[i, i^{*}\right]\right)
\end{aligned}
$$

Since $\sum_{j=1}^{k} c_{\pi(j)}(\sigma)=\left(\sum_{j \in H \cup\{i\}} c_{j}(\sigma)\right)-c_{i^{*}}(\sigma)$ and $\{\pi(1), \ldots, \pi(k)\}=H$ the result follows.

Proposition 2. We are given a spanning tree $\mathcal{T}$ of $G=(V, E)$ rooted at $r$, a spanning tree $T$ induced by a Nash equilibrium $\sigma$ and $i \in N$ (i.e., $i \neq r)$. Let $i^{*}$ be the node of $\mathcal{T}_{i}$ with minimum level in $T$, i.e. $i^{*}=f\left(T, V\left(\mathcal{T}_{i}\right)\right)$. If the triangle inequality holds then

$$
\sum_{j \in V\left(\mathcal{T}_{i}\right)} c_{j}(\sigma)-c_{i^{*}}(\sigma) \leq\left(\operatorname{depth}\left(\mathcal{T}_{i}\right)+1\right) w\left(\mathcal{T}_{i}\right)-w\left(\left[i, i^{*}\right]\right) .
$$

Proof. The proof is done by induction on the depth of $\mathcal{T}_{i}$. If $\operatorname{depth}\left(\mathcal{T}_{i}\right)=0$ (i.e. $i$ is a leaf of $\mathcal{T}$ ) then the result holds since on the one hand $V\left(\mathcal{T}_{i}\right)=\{i\}, i=i^{*}$ and on the other hand $w\left(\mathcal{T}_{i}\right)=0, w\left(\left[i, i^{*}\right]\right)=0$. Remark that the case $\operatorname{depth}\left(\mathcal{T}_{i}\right)=1$ follows from Lemma 2 . So, assume that the result holds for any subtree with depth $d \geq 0$ and consider a subtree $\mathcal{T}_{i}$ with depth $d+1$. Let $M$ be the sons of $i$ in $\mathcal{T}$, i.e. $M=\left\{j \in V\left(\mathcal{T}_{i}\right):[i, j] \in \mathcal{T}\right\}$. For all $s \in M$, the inductive hypothesis gives:

$$
\sum_{j \in V\left(\mathcal{T}_{s}\right)} c_{j}(\sigma)-c_{s^{*}}(\sigma) \leq\left(\operatorname{depth}\left(\mathcal{T}_{s}\right)+1\right) w\left(\mathcal{T}_{s}\right)-w\left(\left[s, s^{*}\right]\right)
$$

where $s^{*}=f\left(T, V\left(\mathcal{T}_{s}\right)\right)$. Using Lemma 2 with $H=\left\{f\left(T, V\left(\mathcal{T}_{s}\right)\right): s \in M\right\}=$ $\left\{s^{*}: s \in M\right\}$, we obtain:

$$
\sum_{j \in H \cup\{i\}} c_{j}(\sigma)-c_{i^{*}}(\sigma) \leq 2\left(\sum_{s \in M} w\left(\left[i, s^{*}\right]\right)\right)-w\left(\left[i, i^{*}\right]\right)
$$




$$
\begin{aligned}
\leq & 2 \sum_{s \in M} w([i, s])+2 \sum_{s \in M} w\left(\left[s, s^{*}\right]\right) \\
& -w\left(\left[i, i^{*}\right]\right)
\end{aligned}
$$

Summing up inequality (20) for all $s \in M$ and adding (21), we get:

$$
\begin{aligned}
\sum_{j \in V\left(\mathcal{T}_{i}\right)} c_{j}(\sigma)-c_{i^{*}}(\sigma) \leq & \sum_{s \in M}\left(\operatorname{depth}\left(\mathcal{T}_{s}\right)+1\right) w\left(\mathcal{T}_{s}\right)+\sum_{s \in M} w\left(\left[s, s^{*}\right]\right) \\
& +2 \sum_{s \in M} w([i, s])-w\left(\left[i, i^{*}\right]\right)
\end{aligned}
$$

On the other hand, using the triangle inequality we deduce that $w\left(\left[s, s^{*}\right]\right) \leq$ $w\left(\mathcal{T}_{s}\right)$ for every $s \in M$. Thus, plugging this last inequality in inequality (22), we get:

$$
\sum_{j \in V\left(\mathcal{T}_{i}\right)} c_{j}(\sigma)-c_{i^{*}}(\sigma) \leq \sum_{s \in M}\left(\operatorname{depth}\left(\mathcal{T}_{s}\right)+2\right) w\left(\mathcal{T}_{s}\right)+2 \sum_{s \in M} w([i, s])-w\left(\left[i, i^{*}\right]\right)
$$

Since $\operatorname{depth}\left(\mathcal{T}_{s}\right) \leq \operatorname{depth}\left(\mathcal{T}_{i}\right)-1,2 \leq \operatorname{depth}\left(\mathcal{T}_{i}\right)+1$ and $w\left(\mathcal{T}_{i}\right)=\sum_{s \in M} w\left(\mathcal{T}_{s}\right)+$ $\sum_{s \in M} w([i, s])$, the result follows from inequality (23). In conclusion, the result holds for $\mathcal{T}_{i}$ and then for all subtree.

Proposition 3. If $d^{*}=2$ then the PoA of the min SST-game is at least 2.

Proof. Consider the complete graph on $N \cup\{r\}$ with $N=\{1, \ldots, n\}$. The weight of the edges is given by $w([r, 1])=1, w([1, i])=1$ for $i=2, \ldots, n$ and the remaining edges have a weight 2 . The triangle inequality holds for this instance and $T^{*}=\{[r, 1]\} \cup\{[1, i]: i=2, \ldots, n\}$ is a minimum weight spanning tree with depth 2 and weight $n$. On the other hand, it is not difficult to see that $T=\{[r, 2],[n, 1]\} \cup\{[i, i+1]: i=2, \ldots, n\}$ with weight $2 n-1$ induces a Nash equilibrium. As $n$ grows the PoA tends to 2 .

\section{Tight examples for Theorem 5.}

Consider an instance with two players 1 and 2 . The weight function is defined as $w([1,2])=w([1, r])=X$ and $w([2, r])=1$ where $X>1$. The strategy profile $\sigma^{*}$ where $\sigma_{1}^{*}=r$ and $\sigma_{2}^{*}=1$ is a social optimum. The maximum degree of a player in the tree induced by $\sigma^{*}$ is 2 (the degree of node 1 ), i.e. $\Delta^{*}=2$. The strategy profile $\sigma$ where $\sigma_{1}=2$ and $\sigma_{2}=r$ is a Nash equilibrium since 1 has no incentive to change her strategy. Therefore the price of anarchy $\mathcal{S}(\sigma) / \mathcal{S}\left(\sigma^{*}\right)=(1+X) / 2 X$ tends towards $1 / 2=1 / \Delta^{*}$ when $X$ tends towards $\infty$. This instance does not satisfy the triangle inequality.

Given $D>1$ and $n \geq D$, we are going to describe an instance $I$ such that:

- I contains at least $n$ players

- $I$ admits a maximum weight spanning tree such that the maximum degree of a player node is $D$, that is $\Delta^{*}=D$

- weights satisfy the triangle inequality 


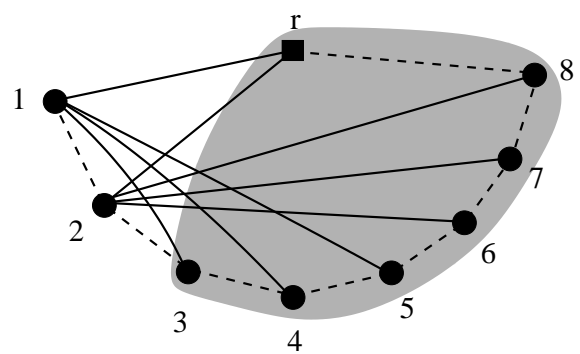

Fig. 4. Instance $I$ when $k=2$ and $\ell=3$. Edges with both endpoints in the grey area have weight 1 and the others have weight $X>1 . T^{*}$ is drawn with solid lines. $T$ is drawn with dashed lines.

- $I$ admits a Nash equilibrium such that $\mathcal{S}(\sigma)=\mathcal{S}\left(\sigma^{*}\right) / D$

An illustration is given in Figure 4.

Let $\ell=D-1$ and $k=\lceil n / D\rceil$. The instance contains a root $r$ and $k(\ell+1)$ player nodes. The weight function is defined as $w([i, j])=1$ if $\{i, j\} \subseteq\{k+$ $1, \ldots, k(\ell+1)\} \cup\{r\}$ and $w([i, j])=X$ otherwise $(X>1)$. The instance satisfies the triangle inequality because there is no triple of nodes $x, y, z$ such that $w([x, y])=w([y, z])=1$ and $w([x, z])=X$.

Let $T^{*}=\{[i, r]: i \in\{1, \ldots, k\}\} \cup\{[i, j]: i \in\{1, \ldots, k\}$ and $k+(i-1) \ell+1 \leq$ $j \leq k+i \ell\} . T^{*}$ is a maximum weight spanning tree (each of its edges has a weight $X)$ with total weight $X k(\ell+1)$. Furthermore, the degree of each node in $\{1, \ldots, k\}$ is $\ell+1$ whereas any node in $\{k+1, \ldots, k(\ell+1)\}$ has degree 1 . Thus, the maximum degree of a player node in $T^{*}$ is $\Delta^{*}=D=\ell+1$.

Let $T=\{[i, i+1]: i \in\{1, \ldots, k(\ell+1)-1\}\} \cup\{[k(\ell+1), r]\}$. The total weight of $T$ is $k(X+\ell)$. Players 1 to $k$ have the largest possible utility $(X)$ so they have no incentive to change their strategy. If a player $i \in\{k+1, \ldots, k(\ell+1)\}$ plays $j \in\{1, \ldots, k\}$ then its utility is $-\infty$ because $i \in \operatorname{visited}(j, r)$. It follows that the strategy profile induced by $T$ is a Nash equilibrium.

We get $\operatorname{PoA} \leq \frac{k(X+\ell)}{X k(\ell+1)}=\frac{1+\ell / X}{\ell+1}$ which tends towards $\frac{1}{\ell+1}=1 / \Delta^{*}$ when $X$ tends towards $\infty$. 